\title{
LIDAR UNCERTAINTY MEASUREMENT EXPERIMENT (LUMEX) - UNDERSTANDING SAMPLING ERRORS
}

\author{
A. Choukulkar ${ }^{1 *}$, W.A. Brewer ${ }^{2}$, R.M. Banta ${ }^{2}$, M. Hardesty ${ }^{1}$, Y. Pichugina ${ }^{1}$, Christoph Senff ${ }^{1}$, S. Sandberg ${ }^{2}$, A. \\ Weickmann $^{1}$, B. Carroll ${ }^{3}$, R. Delgado ${ }^{3}$, A. Muschinski $^{4}$ \\ ${ }^{I}$ Cooperative Institute for Research in Environmental Sciences, Boulder \\ ${ }^{2}$ Chemical Sciences Division, National Oceanic and Atmospheric Administration, Boulder \\ ${ }^{3}$ University of Maryland Baltimore County, Baltimore County \\ ${ }^{4}$ North-West Research Associates, Boulder
}

\begin{abstract}
Coherent Doppler LIDAR (Light Detection and Ranging) has been widely used to provide measurements of several boundary layer parameters such as profiles of wind speed, wind direction, vertical velocity statistics, mixing layer heights and turbulent kinetic energy (TKE). An important aspect of providing this wide range of meteorological data is to properly characterize the uncertainty associated with these measurements.
\end{abstract}

With the above intent in mind, the Lidar Uncertainty Measurement Experiment (LUMEX) was conducted at Erie, Colorado during the period June $23^{\text {rd }}$ to July $13^{\text {th }}, 2014$. The major goals of this experiment were the following:

1. Characterize sampling error for vertical velocity statistics

2. Analyze sensitivities of different Doppler lidar systems

3. Compare various single and dual Doppler retrieval techniques

4. Characterize error of spatial representativeness for separation distances up to $3 \mathrm{~km}$

5. Validate turbulence analysis techniques and retrievals from Doppler lidars

This experiment brought together 5 Doppler lidars, both commercial and research grade, for a period of three weeks for a comprehensive intercomparison study. The Doppler lidars were deployed at the Boulder Atmospheric Observatory (BAO) site in Erie, site of a $300 \mathrm{~m}$ meteorological tower. This tower was instrumented with six sonic anemometers at levels from $50 \mathrm{~m}$ to $300 \mathrm{~m}$ with $50 \mathrm{~m}$ vertical spacing.

A brief overview of the experiment outline and deployment will be presented. Results from the sampling error analysis and its implications on scanning strategy will be discussed.

\section{INTRODUCTION}

Every measurement system has uncertainties associated with it and the coherent Doppler lidar is no exception. Understanding these uncertainties is essential not only to improve the measurement technique, but also to ensure proper interpretation of the observations.

The uncertainties associated with Doppler lidar measurements can be split in to the following components:

1. Instrument error

2. Sampling error

3. Error of spatial representativeness

4. Vector retrieval error

Each of these errors are explain briefly below.

\section{Instrument Error:}

Instrument error or instrument noise can be described as the error associated with making the radial velocity measurement. Instrument error arises due to imperfections of the instrument such as limited spectral resolution of the detector, frequency chirp in the transmitted laser signal, and detector noise, among others (for further details, see: Rye and Hardesty 1993; Dabas et al., 1998; Frehlich 2004).

\section{Sampling Error:}

The sampling error can be divided into two parts: (a) Instrument sampling error and (b) Statistical sampling error. Instrument sampling error occurs due to the finite spatial and temporal resolution of the measurement which results in some scales of 
motion being misrepresented. Statistical sampling error occurs when statistics estimated from measurements do not represent true ensemble estimates.

\section{Error of Representativeness:}

Doppler lidar measurements represent a spatial and temporal average depending on the scanning strategy and retrieval technique used. However, correct interpretation of this spatial and temporal footprint is not trivial and hence introduces an error of representativeness in the measurements.

\section{Vector Retrieval Error:}

Coherent Doppler lidars measure the projection of the wind along the line of sight of the beam known as the line of sight (LOS) velocity or radial velocity. Various single and dual Doppler techniques can be used to estimate the twodimensional wind vector from this radial velocity measurement. The error associated with making this estimation is termed vector retrieval error.

In this presentation, measurements made during LUMEX will be used to present an analysis of the sampling error commonly introduced when estimating vertical velocity variance. This quantity is an important variable in studies related to mass flux transport and pollutant dispersion.

\section{METHODOLOGY}

The Lidar Uncertainty Measurement Experiment (LUMEX) was conducted at the Boulder Atmospheric Observatory (BAO) in Erie CO. The $\mathrm{BAO}$ site is the location of a $300 \mathrm{~m}$ met mast which was instrumented with three-axis sonic anemometers from $50 \mathrm{~m}$ to $300 \mathrm{~m}$ above ground at $50 \mathrm{~m}$ intervals. In addition, two scanning Doppler lidars (one NOAA's High Resolution Doppler Lidar (HRDL) and one University of Maryland Baltimore County (UMBC), Leosphere 200S) and three profiling Doppler lidars were also deployed at various location in and around the BAO facility. The location of the instrumentation is shown in Figure 1.

This experiment had two stages. The first stage was calibration and sampling error evaluation and the second stage was vector retrieval uncertainty and error of spatial representativeness evaluation.

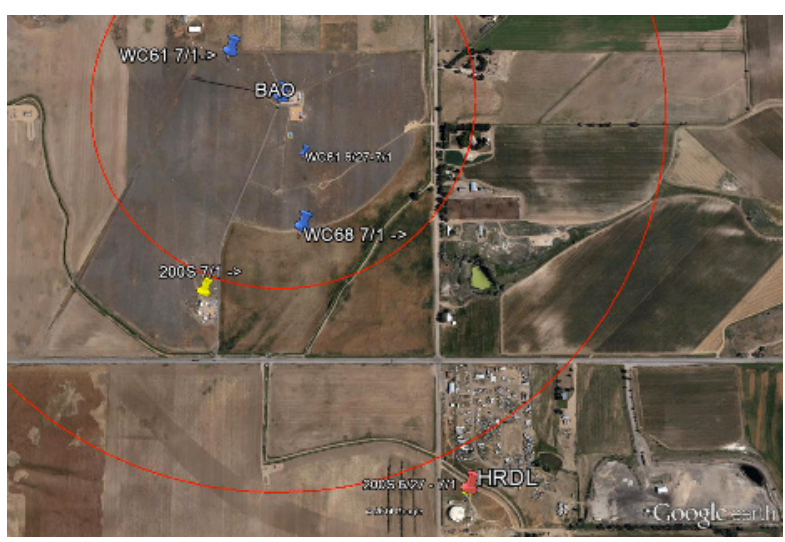

Figure 1. Instrument locations during LUMEX field campaign. The inner red circle is of radius $500 \mathrm{~m}$ centered on the tower while the outer circle is $1000 \mathrm{~m}$ radius centered on the tower.

During the first stage of the experiment, the two scanning Doppler lidars (HRDL and UMBC 200S) were set to stare vertically for 2 full days. In this presentation, the data from these two days will be used to present an analysis on the sampling error for the vertical velocity variance.

\section{RESULTS}

Figure 2 shows the vertical velocity measurements over a three hour period on June $29^{\text {th }}, 2014$. The period is from 1900 UTC to 2200 UTC (1300 hrs to $1600 \mathrm{hrs}$ local time). We see that during this period, the boundary layer is dominated by convective motions and could be called quasistationary state.

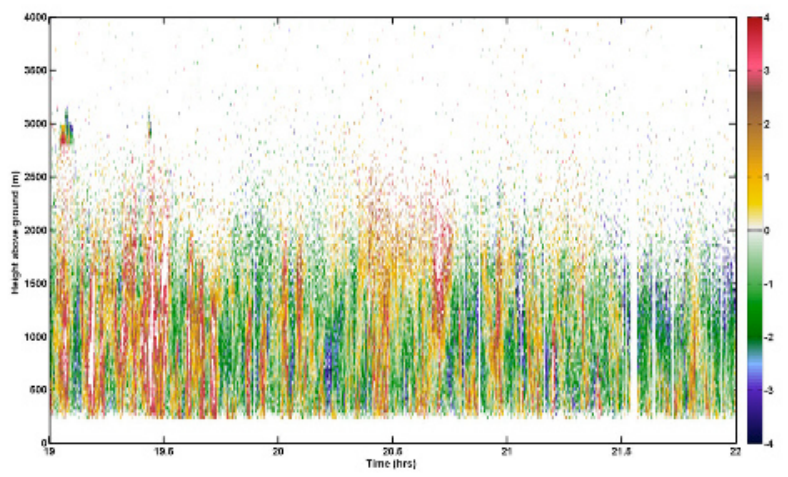

Figure 2. Vertical velocity measurements from June $29^{\text {th }}, 2014$. Warmer colors represent updrafts and cooler colors represent downdrafts.

From this data, vertical velocity variance is estimated for different averaging periods. The sampling error (Lenschow et al., 1994; 2000) for 
the different variance values is also estimated and plotted in Figure 3.

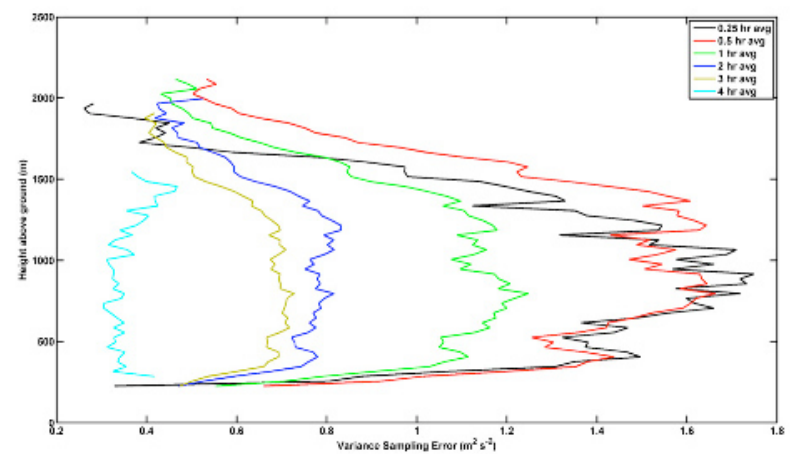

Figure 3. Vertical velocity variance sampling error for different averaging periods ranging from 15 minutes to 4 hours.

As seen from Figure 3, the sampling error reduced steadily as the averaging time is increased. This is consistent with the definition of the ensemble average for a stationary process. However, cases of stationary processes do not always occur in the atmospheric boundary layer. It is common to see large scale features like convective rolls or fronts that have time scales on the order of hours. One such large scale feature is shown in Figure 4.

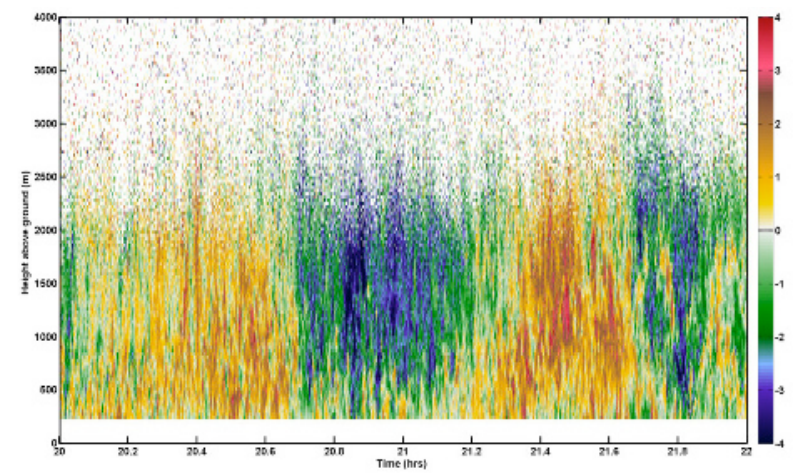

Figure 4. Vertical winds measured on June $30^{\text {th }}$, 2014 from 2000 UTC to 2200 UTC (1400 hrs to $1600 \mathrm{hrs}$ local time)

The sampling errors associated with the vertical velocity variance are shown in Figure 5. It is seen that the smaller time averages have a smaller sampling error associated with them. But this is due to the smaller time averages not capturing the large scale feature completely. It is only when we do a one hour average see the magnitude of our sampling error. Therefore, it is important to note that smaller sampling errors do not always imply that the process has been sampled correctly.

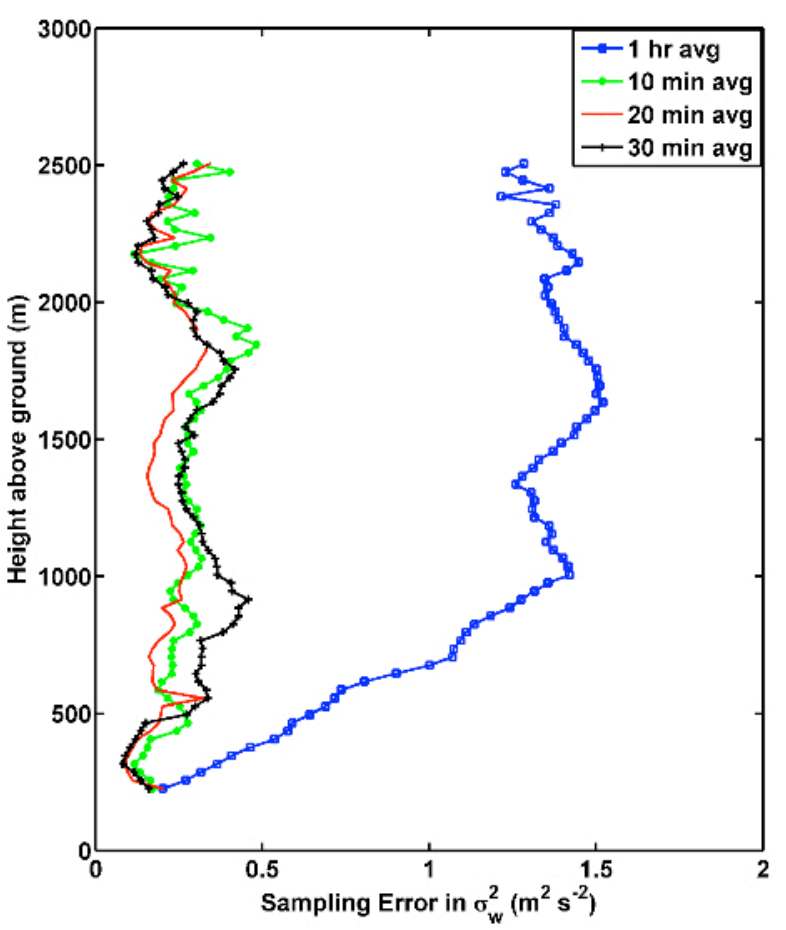

Figure 5. Vertical velocity sampling errors for data collected on June $30^{\text {th }}$, 2014 from 2000 UTC to 2200 UTC.

\section{CONCLUSIONS}

The LUMEX field campaign allows a unique opportunity to evaluate the different sources of uncertainty in Doppler lidar measurements. In this presentation, an overview of the sampling error analysis will be presented. In addition, ongoing work to incorporate this analysis in realtime measurements will be presented.

This sampling error analysis is being extended to horizontal velocity retrievals as well in a subsequent field deployment Experimental Measurement Campaign for Planetary Boundary Layer Instrumentation Analysis (XPIA) conducted in March-April, 2015.

\section{ACKNOWLEDGEMENT}

The authors would like to thank Daniel Wolfe (Physical Sciences Division, NOAA) for his assistance in deployment of this instrumentation at the BAO facility. In addition, the authors would like to thank our collaborators in this field campaign, Julie Lundquist (University of Colorado Boulder) and Keith Barr (Lockheed Martin Coherent Technologies) for bringing their 
instrumentation and lending their expertise in this study.

\section{REFERENCES}

[1] Rye B.J., Hardesty R.M. (1993): Discrete spectral peak estimation in incoherent backscatter heterodyne lidar. II. Correlogram accumulation. IEEE Transactions Geoscience and Remote Sensing, 31, 28-35.

[2] Dabas A.M., Drobinski P., Flamant P.H. (1998): Chirp-induced bias in velocity measurements by a coherent Doppler CO2 lidar. Journal of Atmospheric and Oceanic Technology, 15, 407-415.

[3] Frehlich R. (2004): Velocity Error for Coherent Doppler Lidar with Pulse Accumulation. Journal of Atmospheric and Oceanic Technology, 21, 905-920.

[4] Lenschow D., V. Wulfmeyer, and C. Senff, (2000): Measuring second through fourth-order moments in noisy data. J. Atmos. Oceanic Technol., 17, 1330-1347. 